OPEN ACCESS

Edited by:

Hassane Zouhal,

University of Rennes 2 - Upper

Brittany, France

Reviewed by:

Carlo Capelli,

University of Verona, Italy

Claire Tourny,

Université de Rouen, France

*Correspondence:

Gernot O. Hering

gernot.hering@uni-konstanz.de

Specialty section: This article was submitted to Exercise Physiology, a section of the journal

Frontiers in Physiology

Received: 15 February 2021

Accepted: 31 March 2021

Published: 26 April 2021

Citation:

Hering GO and Stepan J (2021) The

Maximal Lactate Steady State

Workload Determines Individual

Swimming Performance.

Front. Physiol. 12:668123.

doi: 10.3389/fphys.2021.668123

\section{The Maximal Lactate Steady State Workload Determines Individual Swimming Performance}

\author{
Gernot O. Hering ${ }^{1 *}$ and Jens Stepan ${ }^{1,2}$ \\ ${ }^{1}$ Department of Sport and Health Science, University of Konstanz, Konstanz, Germany, ${ }^{2}$ Department of Obstetrics and \\ Gynaecology, Paracelsus Medical University, Salzburg, Austria
}

The lactate threshold (LT) and the strongly related maximal lactate steady state workload (MLSS $\left.S_{w}\right)$ are critical for physical endurance capacity and therefore of major interest in numerous sports. However, their relevance to individual swimming performance is not well understood. We used a custom-made visual light pacer for real-time speed modulation during front crawl to determine the LT and MLSS in a single-exercise test. When approaching the $\mathrm{LT}$, we found that minute variations in swimming speed had considerable effects on blood lactate concentration ([La-]). The LT was characterized by a sudden increase in [La], while the MLSS ${ }_{w}$ occurred after a subsequent workload reduction, as indicated by a rapid cessation of blood lactate accumulation. Determination of the MLSS by this so-called "individual lactate threshold" (ILT)-test was highly reproducible and valid in a constant speed test. Mean swimming speed in 800 and 1,500 m competition (S-Comp) was 3.4\% above MLSS level and S-Comp, and the difference between S-Comp and the $\mathrm{MLSS}_{\mathrm{W}}\left(\Delta \mathrm{S}\right.$-Comp/MLSS ${ }_{\mathrm{W}}$ ) were higher for long-distance swimmers (800-1,500 m) than for short- and middle-distance swimmers (50-400 m). Moreover, $\Delta$ S-Comp/MLSS varied significantly between subjects and had a strong influence on overall swimming performance. Our results demonstrate that the $\mathrm{MLSS}_{\mathrm{w}}$ determines individual swimming performance, reflects endurance capacity in the sub- to supra-threshold range, and is therefore appropriate to adjust training intensity in moderate to severe domains of exercise.

Keywords: lactate threshold, maximal lactate steady state, swimming, performance testing, exercise physiology

\section{INTRODUCTION}

Changes in physical endurance capacity require precise adaptation of various physiological processes. The exact knowledge of the exercise associated stimulus/response pattern, and the availability of reliable and valid diagnostic systems are prerequisites to induce particular homeostatic perturbations and to implement successful training interventions (Fluck and Hoppeler, 2003). Frequency, duration and volume are the major parameters for controlling training load. However, selective adjustment of training intensity is the most potent trigger of endurance capacity specification (Bishop et al., 2019; Jamnick et al., 2020).

At some point, a steadily increasing workload (WL) will lead to rapid arterial lactate accumulation. This so-called lactate threshold (LT; Brooks, 1985a) is closely related to the maximal lactate steady state workload $\left(M_{L S S}\right)$, the highest WL with equilibrium between lactate production and removal and thus constant arterial lactate concentration $\left[\mathrm{La}^{-}\right]$(Donovan and Brooks, 1983; 
Brooks, 1985a,b; Heck et al., 1985; Stanley et al., 1985). For the following reasons, others and we suggest the MLSS major submaximal parameter for individual training intensity recommendations (Billat et al., 2003; Faude et al., 2009; Hering et al., 2018; Jamnick et al., 2020): First, the MLSS $_{\mathrm{W}}$ is highly reproducible, valid, and objective across endurance sports (Hering et al., 2018; Jamnick et al., 2020). Second, it has been repeatedly shown that exercise in the $\mathrm{MLSS}_{\mathrm{W}}$ range induces key neuromuscular and metabolic adaptations (Bergman et al., 1999; Dubouchaud et al., 2000; Messonnier et al., 2013; Figueiredo et al., 2014; de Araujo et al., 2015; Hering et al., 2018).

At the $\mathrm{MLSS}_{\mathrm{W}}$, minute WL increments cause a rapid increase in $\left[\mathrm{La}^{-}\right]$, which is very likely due to an imbalance between lactate production and removal within the complex shuttle network of lactate metabolism (Donovan and Brooks, 1983; Brooks, 1985b; Stainsby and Brooks, 1990; Messonnier et al., 2013). An underlying neuromuscular mechanism is increased activity in motor units with high lactate efflux. If activity in lactate-consuming motor units remains unchanged, this triggers rapid arterial lactate accumulation in relation to power output. In addition, changes in the intra- and inter-muscular innervation, muscle-fiber recruitment pattern, fatigue, and in molecular signaling cascades contribute to perturbations of lactate homeostasis at the MLSS $_{\mathrm{W}}$ (Hering et al., 2018).

The traditional method of $\mathrm{MLSS}_{\mathrm{W}}$ determination requires several tests on different days and is therefore time-consuming and inappropriate for daily use (Heck et al., 1985; Figueiredo et al., 2014; Pelarigo et al., 2017; Jamnick et al., 2020). To circumvent these problems, we recently introduced a singleexercise test in running and cycling (Hering et al., 2018). Here, we have adapted the test algorithm for swimming and can reliably determine the $\mathrm{MLSS}_{\mathrm{W}}$ in less than $1 \mathrm{~h}$. Our data not only recapitulate a remarkably fine-tuned regulation of lactate metabolism at the $\mathrm{MLSS}_{\mathrm{W}}$ in swimming, but also provide evidence that the $\mathrm{MLSS}_{\mathrm{W}}$ reflects physical endurance capacity in ranges above the MLSS ${ }_{\mathrm{W}}$. Consequently, we suggest the MLSS as an important submaximal parameter for the precise adjustment of training stimuli in moderate to severe domains of exercise.

\section{MATERIALS AND METHODS}

\section{Subjects}

Fifty-five single-exercise swimming tests [50 amateur athletes with regular participation in competitions up to national championships aged $11-32$ years (19 \pm 0.74 years, 27 females)] were selected for ILT and MLSS $_{\mathrm{W}}$ determination. Each participant gave written, informed consent after being provided a detailed description of the study requirements and protocols. The experimental procedures were approved by the University of Konstanz Institutional Review Board and were performed in accordance with the ethical standards of the Government of Baden Württemberg. All subjects were healthy and avoided heavy exercise and maintained a normal diet at least 2 days before testing.

\section{Pacing System}

The visual light pacing system consisted of 25 interconnected polycarbonate tubes, each with four light-emitting diodes. The 100 red lights could be switched on and off individually via a microcontroller connected to a PC (control box for swimming speed, Figure 1). A waterproof power cable and a steel cable ensured a constant distance of $25 \mathrm{~mm}$ between the diodes (Figure 1). The software-controlled, portable pacing system was located on the pool floor and the swimmer followed the continuously moving light strip (Figure 1).

\section{Study Protocol of Swim Tests}

The basic protocol for the so called individual lactate threshold (ILT)-test was identical to previous treadmill and bicycle ergometer tests (Hering et al., 2018; for detailed explanation see below, and Figures 2A,B, 3). The ILT-test consists of five distinct

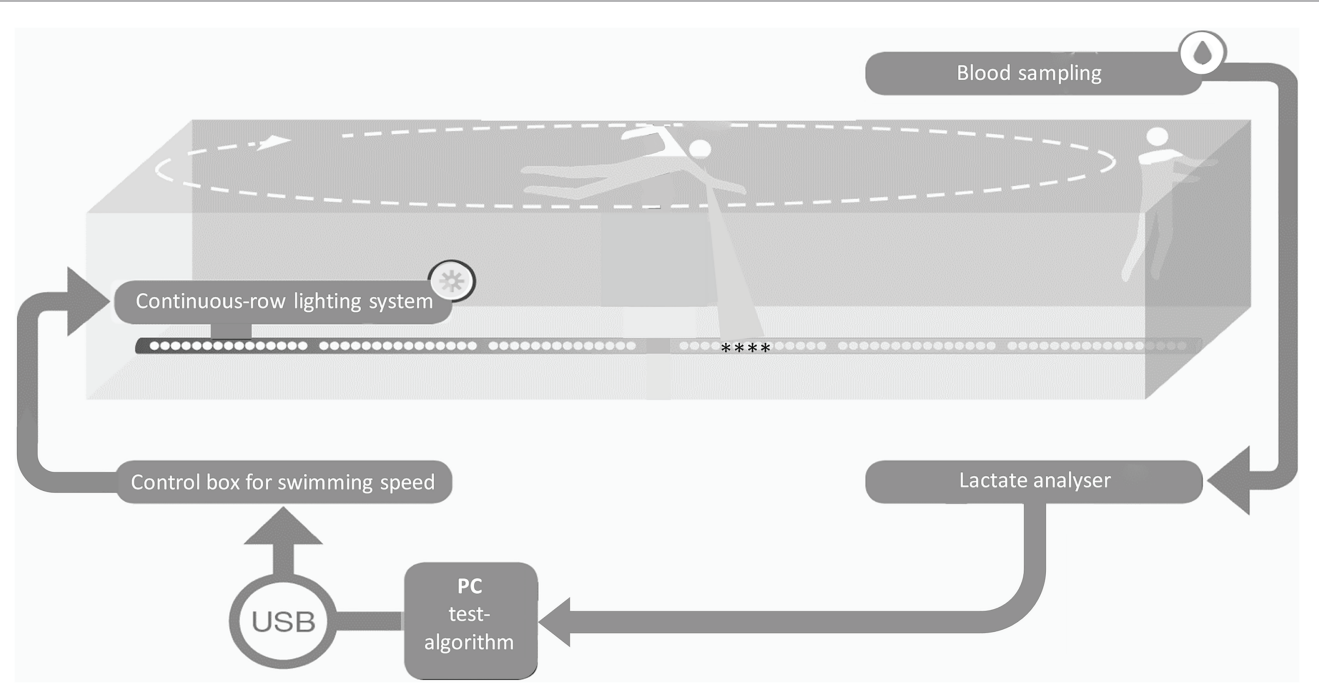

FIGURE 1 | Experimental arrangement of the individual lactate threshold-test in swimming. 
stages: (i) warm-up ( $\mathrm{W}_{\mathrm{UP}}$ ), (ii) threshold adaptation (TA), (iii) fine threshold adaptation (FTA), (iv) lactate threshold detection (LTD) by means of two threshold criteria (TC), followed by (v) a minimal workload reduction for verification of the $\mathrm{MLSS}_{\mathrm{W}}$ (Figures 2A,B, 3, 4A,B). It is important to note that the ILT-test does not have a fixed number of stages and the swimming speed is set in relation to the $\left[\mathrm{La}^{-}\right]$from the previous step.

For the present study, all 55 datasets were retrospectively analyzed with the latest TC. The TC were refined over several years to optimize the test procedure (e.g., faster FTA), the results however, have not changed. Each subject performed a warm-up in a $25 \mathrm{~m}$ swimming pool at a self-chosen pace $\left(\mathrm{S}_{\text {Sin }}\right)$, followed by a 60 -s period to calibrate the visual light pacing system. The initial swimming speed was set $0.025 \mathrm{~m} / \mathrm{s}$ above $\mathrm{S}_{\mathrm{Sin}}$. The finetuning toward the LT was achieved by means of several steps with slight WL increments until a TC was realized (Figures 2A,B, 3). Every WL increment/reduction was set in accordance to lactate concentration from the previous step (Figures $\mathbf{2 A , B}, \mathbf{3}$ ). We favored a 3-4-min step duration to ensure a metabolic and blood circulation adaptation phase of at least $3 \mathrm{~min}$ after a potential WL variation (Weltman et al., 1990; Bentley et al., 2007). The blood sample was taken when the swimmer reached the end of the pool after a step duration of at least $180 \mathrm{~s}$. [ $\left.\mathrm{La}^{-}\right]$was measured [Arkray Lactate Pro ${ }^{\circledR}$ 1710, sampling volume $5 \mu \mathrm{l}$, measuring time $60 \mathrm{~s}$, coefficient of variation $=3 \%$ (Pyne et al., 2000; Baldari et al., 2009; Tanner et al., 2010; Mamen, 2014)], after swimming was stopped for approximately $15 \mathrm{~s}$ to collect capillary blood samples from the hyperemirized earlobe (Finalgon ${ }^{\circledR}$, Sanovi-Aventis Germany GmbH, Frankfurt am Main, Germany; Dassonville et al., 1998; Feliu et al., 1999). Afterward, the test
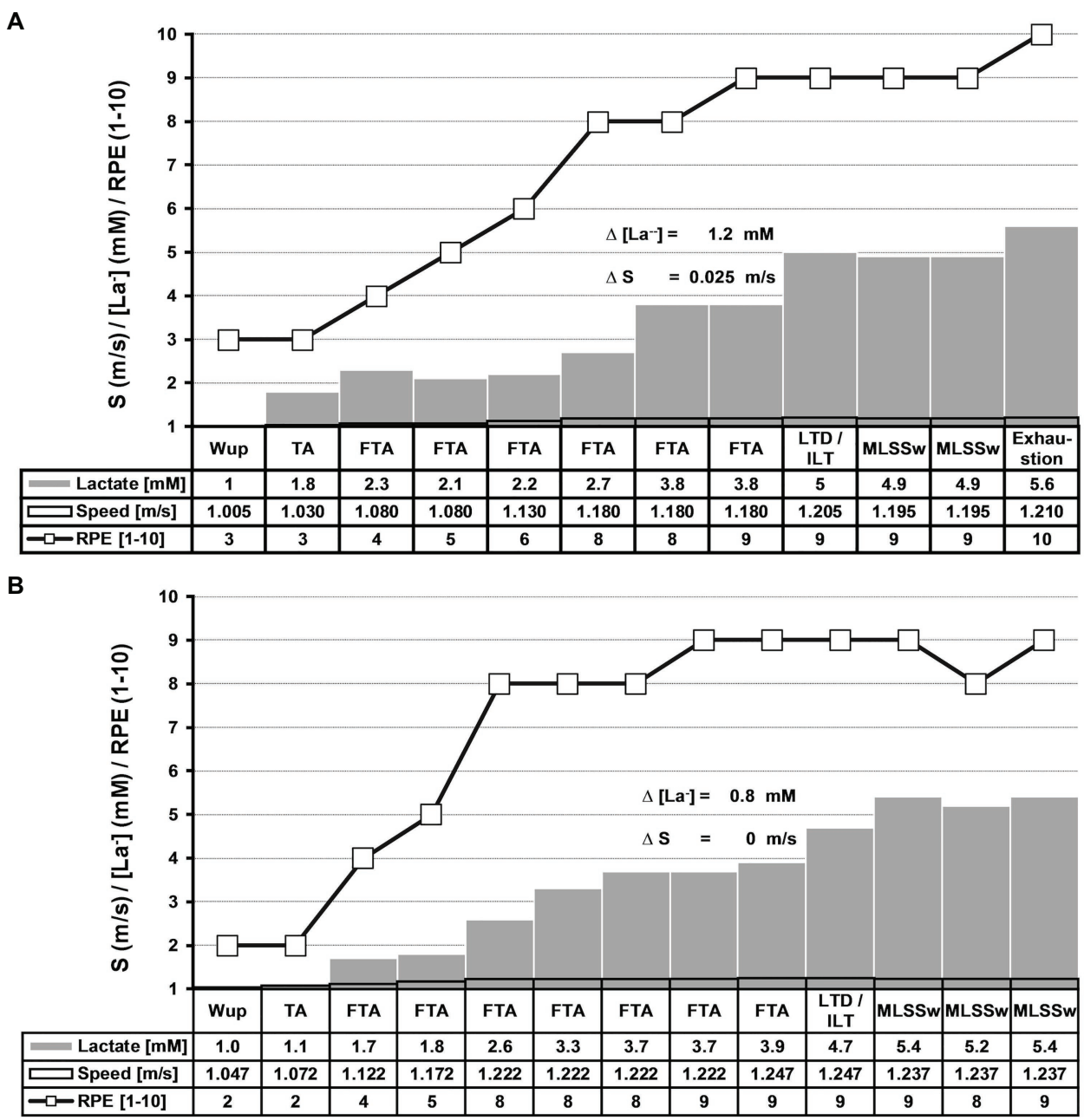

FIGURE 2 | Experimental protocols of two typical ILT-tests. (A) LT detection with threshold criterion 1 (TC1) and (B) with threshold criterion 2 (TC2; Figure 3). The basic experimental protocol is identical on the treadmill, bicycle ergometer, and for swimming (for detailed information, see Figures 4A,B; Hering et al., 2018). After warm-up (WuP), threshold adjustment (TA), and fine threshold adjustment (FTA; see section "Material and Methods"), a step-like blood lactate accumulation occurred at the individual lactate threshold (ILT; $\left.\Delta\left[\mathrm{La}^{-}\right]\right)$after 1 (TC1; A) or after up to six steps (TC2; B). Immediately or one increment after a comparable slight workload reduction $(0.01-0.015 \mathrm{~m} / \mathrm{s})$, the lactate accumulation slowed down, indicating the maximal lactate steady state workload (MLSS $)$. Real-time workload adjustments were set in accordance to blood lactate level in the previous exercise period, explaining the variable test durations even intraindividually (data not shown). [La-], blood lactate concentration; RPE, rate of perceived exertion; S, speed. 
was continued at the pre-rest speed until the blood sample was analyzed.

Figures 2A,B, 3 depict the algorithms for the TA, the FTA, and the LTD. After detection of the LT, swimming speed was slightly reduced $(0.01-0.015 \mathrm{~m} / \mathrm{s})$ to stop lactate accumulation and to verify the MLSS $_{\mathrm{W}}$. Participants were instructed to rate exercise intensity on a scale from 1 to 10,1 is defined as very light and 10 as the maximum load (Borg, 1982).

The test-protocol, data analyses, and documentation were executed automatically by custom written software. The digitized speed data were averaged across $5 \mathrm{~s}$ intervals and stored together with lactate levels, RPE, anthropometric, and time dependent data.

\section{Constant Speed-Test}

The validity and reproducibility were assessed in a constant speed (CS)-test with 13 regional competitive swimmers/triathletes aged $19-32$ years $(26.2 \pm 1.1$ years, 5 females $)$. Here, the initial speed was set $0.02 \mathrm{~m} / \mathrm{s}$ below the previously determined swimming speed at the MLSS $\mathrm{W}_{\mathrm{W}}$ and increased by $0.01 \mathrm{~m} / \mathrm{s}$ if the $\left[\mathrm{La}^{-}\right]$was constant $\left(\Delta\left[\mathrm{La}^{-}\right]<0.3 \mathrm{mM}\right)$ in two consecutive 3-min steps. When a TC was realized, swimming speed was reduced by $0.01 \mathrm{~m} / \mathrm{s}$ to stop or, at least, to stabilize the increase in $\left[\mathrm{La}^{-}\right]$. The test consisted of at least 10 steps with a break of $15 \mathrm{~s}$ in between. The RPE was recorded simultaneously.

\section{Validation of the Study Protocol in Swimming Competitions}

Seven to fourteen days before an official swimming competition (800 m women/1500 m men), all 22 subjects aged 13-23 years (17.3 \pm 0.92 years, 13 women/9 men, swim training/strength training 8.5/2.4 h/week) conducted an ILT-test as described above. According to their own statements and based on their

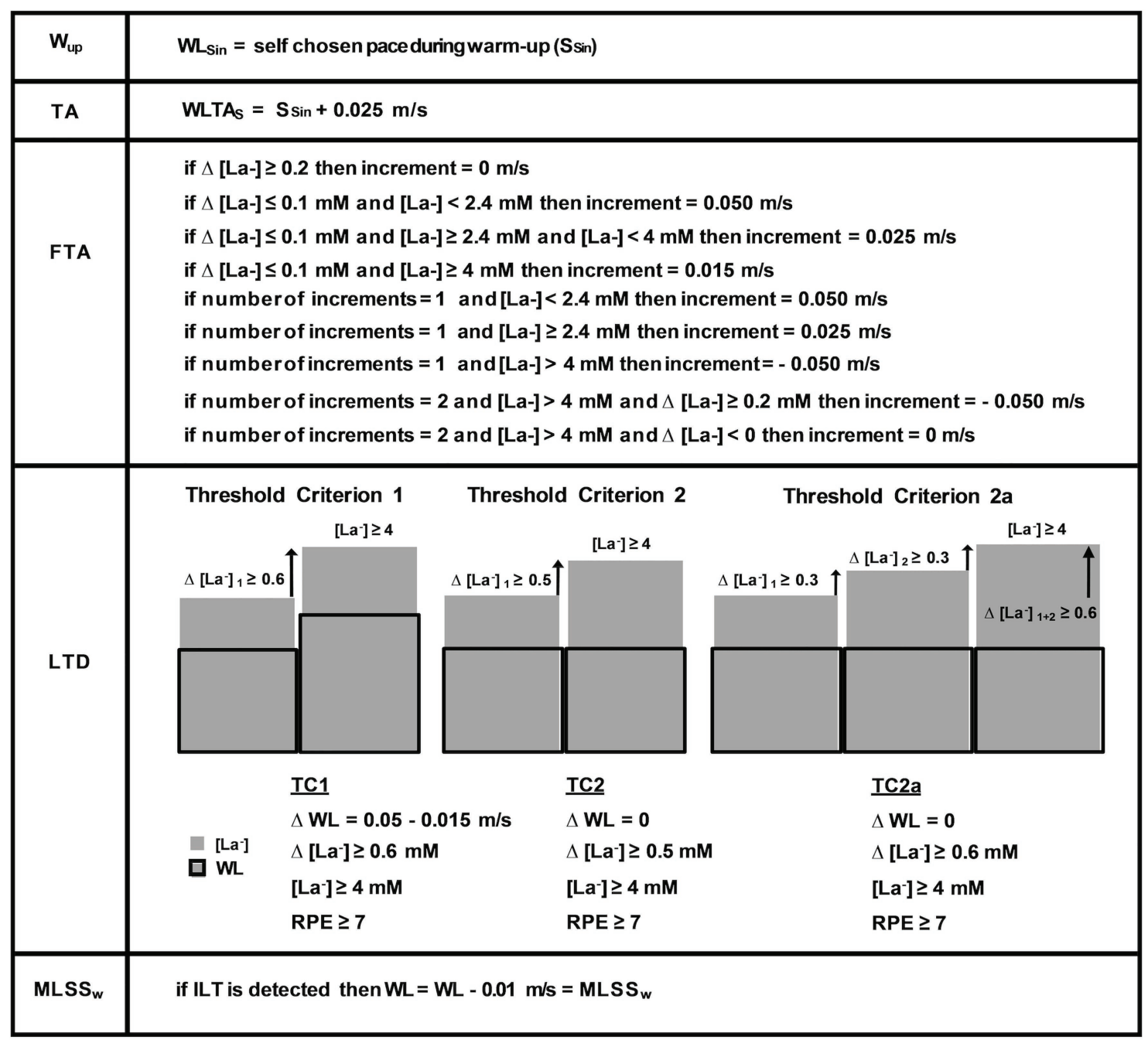

FIGURE 3 | ILT-test algorithms. Swimming pace ( $\left.\mathrm{S}_{\mathrm{Sin}}\right)$ during warm-up ( $\left.\mathrm{W}_{\mathrm{UP}}\right)$ was self-chosen. The fine threshold adjustment (FTA) was executed in accordance to test duration and blood lactate dynamics. The current threshold criteria (TC) 1, 2 and 2a for determination of the individual lactate threshold (ILT), have been developed into their current form by continuously incorporating ILT-test data. After determination of the individual lactate threshold (ILT), the lactate accumulation usually slowed down after a slight workload reduction indicating the MLSS $\mathrm{W}\left[\mathrm{La}^{-}\right]$, blood lactate concentration; MLSS $\mathrm{w}_{\mathrm{w}}$ maximal lactate steady state workload; RPE, rate of perceived exertion; and WLTAs, initial workload adaptation. 
A
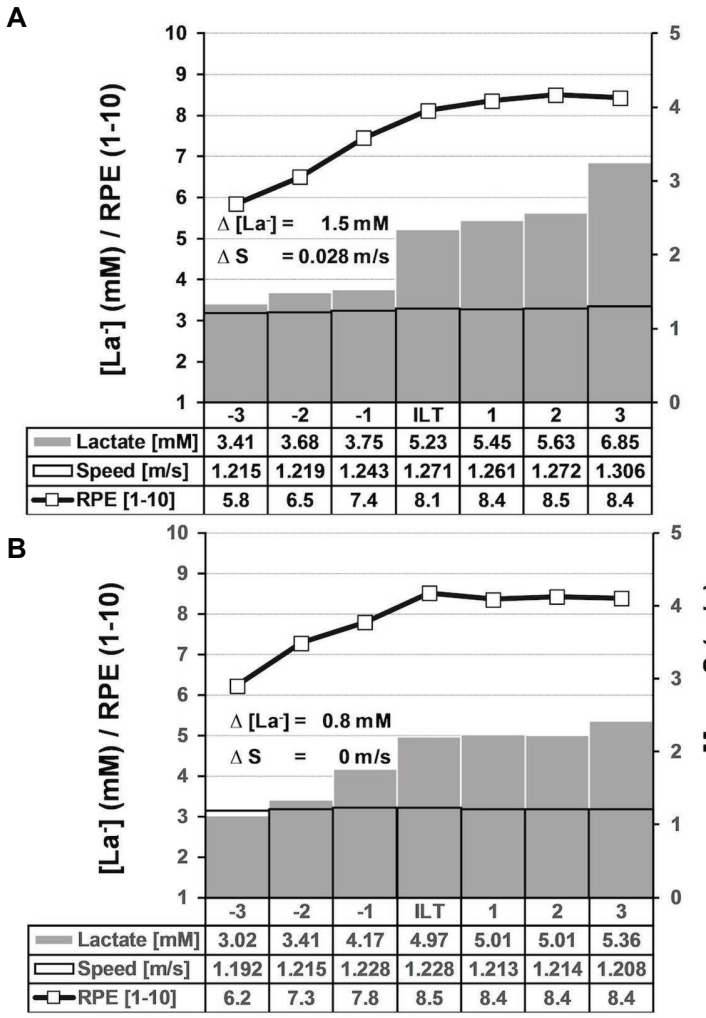

C

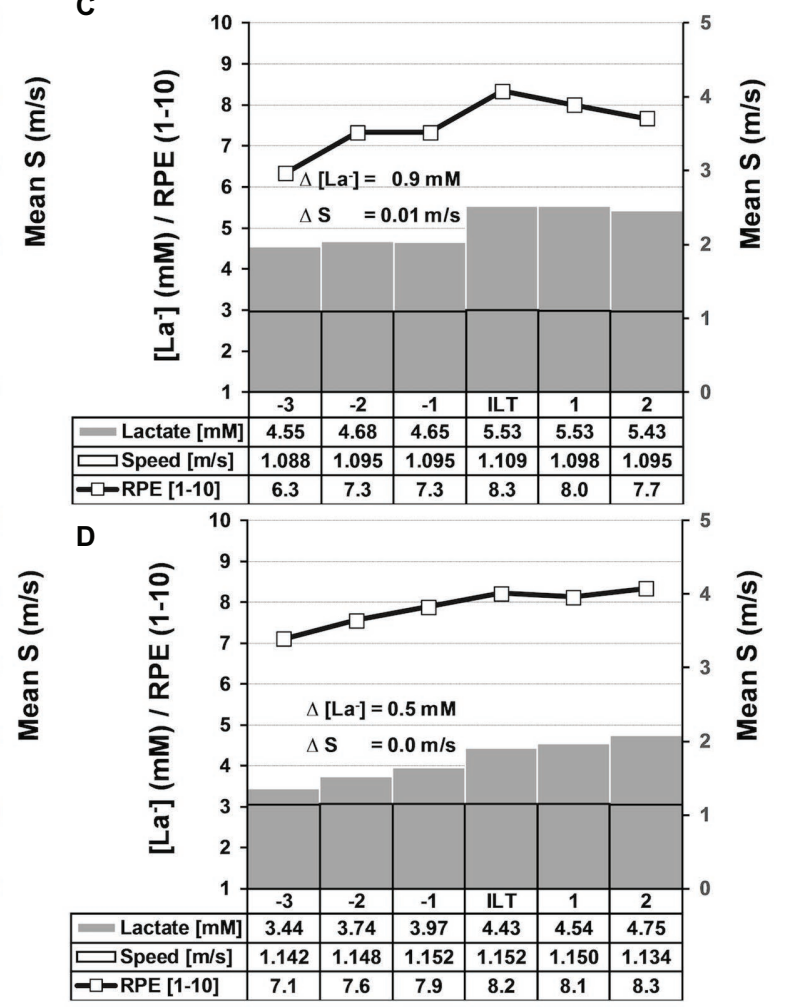

FIGURE 4 | Summary of (individual lactate threshold) ILT-tests and constant speed (CS)-tests. (A,B) Summary of ILT-tests that met threshold criterion 1 (A, $n=18$ ) or $2(\mathbf{B}, n=37)$. Group mean values are shown for blood lactate concentration $(\mathrm{mM})$, speed $(\mathrm{m} / \mathrm{s})$, and RPE (1-10). Note that the step-like lactate accumulation at the ILT in response to a minute increase in swimming speed. (A) Threshold criterion 1: $\Delta\left[\mathrm{La}^{-}\right]=1.5 \pm 0.237 \mathrm{mM}, \Delta \mathrm{S}=0.028 \pm 0.003 \mathrm{~m} / \mathrm{s}$. (B) Threshold criterion 2 : $\Delta\left[\mathrm{La}^{-}\right]=0.8 \pm 0.065 \mathrm{mM}$. (C/D) Summary of (CS)-tests that met threshold criterion $1(\mathbf{C}, \mathrm{n}=4)$ or $2(\mathbf{D}, \mathrm{n}=9)$. Group mean values are shown for blood lactate concentration $(\mathrm{mM})$, Speed $(\mathrm{m} / \mathrm{s})$, and RPE (1-10). Note that the step-like lactate accumulation at the ILT in response to a minute increase in swimming speed. (C) Threshold criterion 1: $\Delta\left[\mathrm{La}^{-}\right]=0.9 \pm 0.17 \mathrm{mM}, \Delta \mathrm{S}=0.01 \mathrm{~m} / \mathrm{s}, n=4$. (D) Threshold criterion 2: $\Delta\left[\mathrm{La}^{-}\right]=0.5 \pm 0.05 \mathrm{mM}$. Data are mean $\pm \mathrm{SE}$. [La- $]$, arterial lactate concentration; RPE, rate of perceived exertion; S, speed.

training and competition history, 10 athletes, aged 14-31 years (17.9 \pm 1.6 years, nine females) were defined as long-distance swimmers. Twelve subjects, aged 13-26 (16.8 \pm 1.1 , six females) were assigned to the short-/middle-distance group. The group assignment based on the official FINA regulations (FINA, F.I.d.N., 2021). No information about the ILT-test results was given to the athletes before competition. The average swimming speed during competition was compared to ILT-test results. For the analysis of individual swimming performance, four male swimmers aged $17-22$ years (19.8 \pm 1 years) completed a full set of Olympic distances (50, 100, 200, 400, 1,500 m) and $800 \mathrm{~m}$ within 3-14 days after the ILT-test (Figure 5).

\section{Statistical Analysis}

Statistical analyses were run in SPSS 24.0 (IBM). Data were analyzed by two-tailed paired and unpaired $t$-tests as appropriate. The F-test was used to test for equality of variances. Correlations between variables were assessed using Pearson's correlation coefficient $(r)$. Coefficient of variation (CV) was calculated for the ILT-/CS-test comparison. Data are shown as the mean $\pm \mathrm{SE}$ unless otherwise stated. $p<0.05$ were considered as statistically significant.

\section{RESULTS}

For running and cycling, we recently demonstrated that the equilibrium between lactate production and elimination and, thus, the MLSS ${ }_{\mathrm{W}}$ can be disturbed by minute changes in exercise intensity $( \pm 0.1 \mathrm{~m} / \mathrm{s} / 7 \mathrm{~W}$; Hering et al., 2018). Here, the athletes could implement such small changes in swimming speed by means of a visual light pacer mounted on the pool floor (Figure 1; see also section "Material and Methods"). In this way, we have determined LT and MLSS ${ }_{\mathrm{W}}$ in 55 "individual lactate threshold-tests" (ILT-tests) in swimmers (Figures 4A,B).

We previously defined two threshold criteria (TC) for treadmill and bicycle ergometer tests based on an algorithm that has been developed into its current form by continuously incorporating ILT-test data (Hering et al., 2018). We applied the same procedure on swimming tests and, again, uncovered two scenarios of blood lactate accumulation at the LT (Figures 2A,B, 3; Hering et al., 2018). Either the arterial blood lactate concentration $\left[\mathrm{La}^{-}\right]$raised more than $0.5 \mathrm{mM}$ in the exercise period directly after a WL increment (TC1) or it raised more than $0.4 \mathrm{mM} \mathrm{1-6}$ steps after the initial WL increment (TC2; Figures 2A,B, 3, 4A,B). Both, TC1 and TC2 


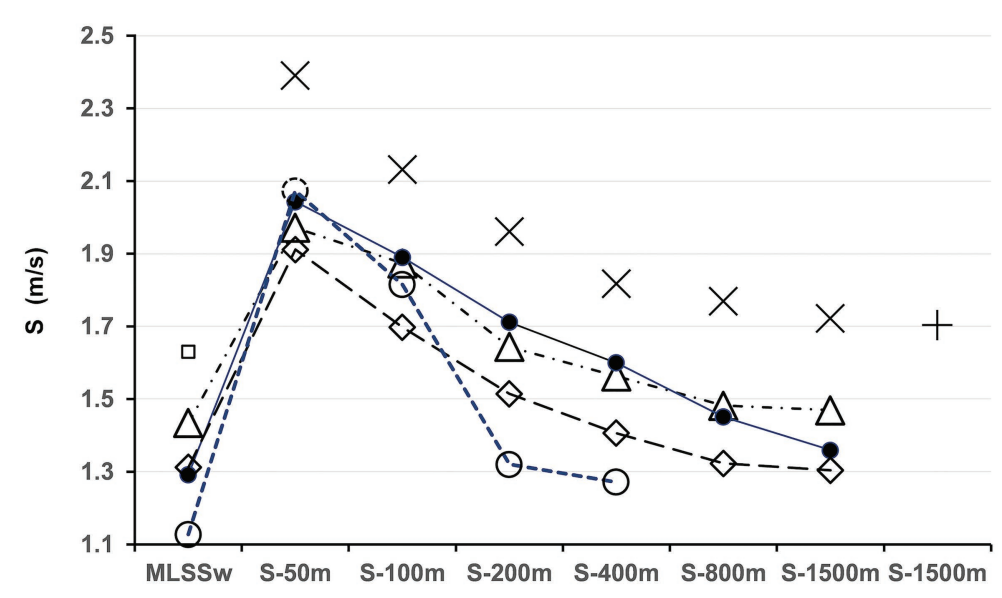

FIGURE 5 | Comparison of swimming performance in relation to maximal lactate steady state workload (MLSS ${ }_{w}$ ) across Olympic distances. $\mathbf{\Delta}=$ Test person (TP) 1 , $\boldsymbol{0}=$ TP 2, $\diamond=$ TP3, O = TP4, $\square$ = estimated MLSS $w$ Australian National Team 1996, according to Pyne et al. (2001), + = world record 1994 (Kieren Perkins, Australia), $X=$ current world records (FINA, F.I.d.N., 2021). TP1 had the highest MLSS , resulting in comparatively good results across all distances. TP4 was slightly faster at $50 \mathrm{~m}$, but slowed down rapidly at longer distances. Note that TP2 performed close to the $\mathrm{MLSS}_{\mathrm{w}}$ in the $1,500 \mathrm{~m}$ race $\left(\Delta \mathrm{S}-\mathrm{Comp}_{\mathrm{MLSS}}=-0.008 \mathrm{~m} / \mathrm{s}\right) . \Delta \mathrm{S}-\mathrm{Comp} /$ MLSS $_{w}$ for TP1/TP3 was moderate $(0.035 / 0.067 \mathrm{~m} / \mathrm{s})$ with a relatively high $\mathrm{MLSS}_{w}$ for TP1 and a relatively low MLSS for TP3 $(1.434 / 1.387 \mathrm{~m} / \mathrm{s}$; for a detailed analysis see section "Discussion"). Since MLSS data from world record holders were not available, we estimated the values using data from Pyne et al. (2001). Although these calculations are certainly not entirely accurate, they clearly demonstrate the large gap between MLSS w1996 $_{\text {and }}$ the 1,500 m world record in 1994. S, speed.

were characterized by a steep increase in $\left[\mathrm{La}^{-}\right]$, which occurred between absolute lactate levels of 1.8-7 mM (Figures 4A,B). The mean increase in $\left[\mathrm{La}^{-}\right]$was $1.1 \pm 0.91 \mathrm{mM}$ and the maximum increase was $5.1 \mathrm{mM}$ (Figures $4 \mathbf{A}, \mathbf{B}$ ). In 4 out of 55 tests, the increase in $\left[\mathrm{La}^{-}\right]$did not reach $0.5 \mathrm{mM}$, but raised slowly in at least three successive steps at constant swimming speed (TC2a; Figure 3). After ILT detection, a slight workload reduction $(0.01-0.015 \mathrm{~m} / \mathrm{s})$ stopped lactate accumulation and was used for $\mathrm{MLSS}_{\mathrm{W}}$ verification (Figures 2A,B, 3, 4A,B). The rate of perceived exertion (RPE) raised or kept constant at high subjective fatigue levels after reaching the ILT (Figures $\mathbf{2 A , B}, \mathbf{4 A}, \mathbf{B}$ ).

Notably, rapid blood lactate accumulation $(>0.4 \mathrm{mM}, \mathrm{TC} 1 / 2)$ occurred within six steps at constant pace, after swimming speed was increased very slightly $(0.01 \mathrm{~m} / \mathrm{s})$. We next evaluated the impact of higher increments on lactate dynamics. While increments of 0.015 and $0.025 \mathrm{~m} / \mathrm{s}$ do not differ from $0.01 \mathrm{~m} / \mathrm{s}$, a step of $0.05 \mathrm{~m} / \mathrm{s}$ provoked another significant lactate accumulation (Figure 6A). These data show an extremely sensitive regulated lactate metabolism in swimming and indicate that minute changes in workload can rapidly unbalance lactate homeostasis.

To examine the validity and reproducibility of the ILT-test, we verified the results in a CS-test of at least 30 min near the MLSS $_{\mathrm{W}}$ (see section "Material and Methods"). Swimming speed at the MLSS correlated well between ILT-tests and CS-tests (Figure 6B). The minimal difference in average swimming speed at the MLSS between the two test protocols $(0.002 \mathrm{~m} / \mathrm{s})$ was below the smallest workload increment used in the ILT-tests $(0.01 \mathrm{~m} / \mathrm{s}$; Figure $6 B)$. In addition, the test-retest coefficient (CC, 0.997 ) and the coefficient of variation $(\mathrm{CV}, 0.49 \%)$ are below the values known from the literature for day to day variability of the so-called "Gold Standard Method" (Hauser et al., 2013; $\mathrm{CC}=0.98, \mathrm{CV}=3 \%$; Batschelet, 2004; $\mathrm{CV}=0.77 \%)$.
This demonstrates the high reliability and validity of the ILT-test in accessing the MLSSw in swimming. As already shown for the ILT-test, increasing swimming speed by only $0.01 \mathrm{~m} / \mathrm{s}$ resulted in a pronounced change in lactate kinetics (Figures 4C,D).

To evaluate the physiological relevance of the ILT-test in swimming, we compared the test results with data obtained from long distance competitions $(800 / 1500 \mathrm{~m})$. All swimmers performed the ILT-test, followed by a competition after at least 7 days of recovery. In accordance with our recent study on half-marathon runners (Hering et al., 2018), swimming speed in ILT-tests correlated with swimming speed in competition (Figures 7A,C). However, while the average running speed in a half-marathon was $5.2 \%$ below threshold speed (Hering et al., 2018), the swimmers performed 3.4\% above their MLSS $_{W}$ (S-Comp vs. MLSS $_{\mathrm{W}}$, Figures 7A,C). When the same analysis was applied separately for short-/middle-distance (S-Comp-SM, 50-400 m) and long-distance athletes (S-Comp-L, 800/1500 m), a $\Delta \mathrm{S}$-Comp/MLSS $\mathrm{W}_{\mathrm{W}}$ only occurred in the long-distance group (S-Comp-L vs. MLSS $\mathrm{W}_{\mathrm{W}} \mathrm{L}$, Figure 7B). Finally, we found a close relationship between S-Comp and $\Delta \mathrm{S}$-Comp/MLSS ${ }_{\mathrm{W}}(\Delta \mathrm{S}$-Comp/ $\mathrm{MLSS}_{\mathrm{W}}$ vs. S-Comp, Figure 7D), which is more pronounced in long-distance swimmers ( $\Delta$ S-Comp-L/MLSS $\mathrm{W}_{\mathrm{W}} \mathrm{L}$ vs. $\Delta$ S-Comp-SM/MLSS $\mathrm{W}_{\mathrm{W}}-\mathrm{SM}$, Figure 7B). This finding demonstrates the major role of supra-threshold capacity in swimming and very likely determines swimming performance on longer distances (S-Comp-L vs. S-Comp-SM, Figure 7B).

\section{DISCUSSION}

Along with biochemical and morphological adaptation, successful training stimuli integrate dimensions of time, movement 


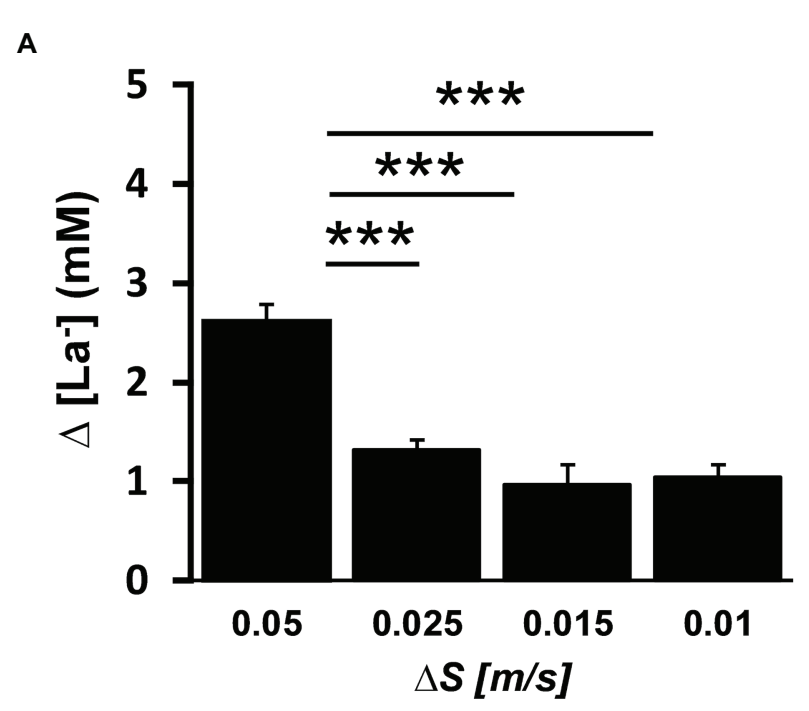

B

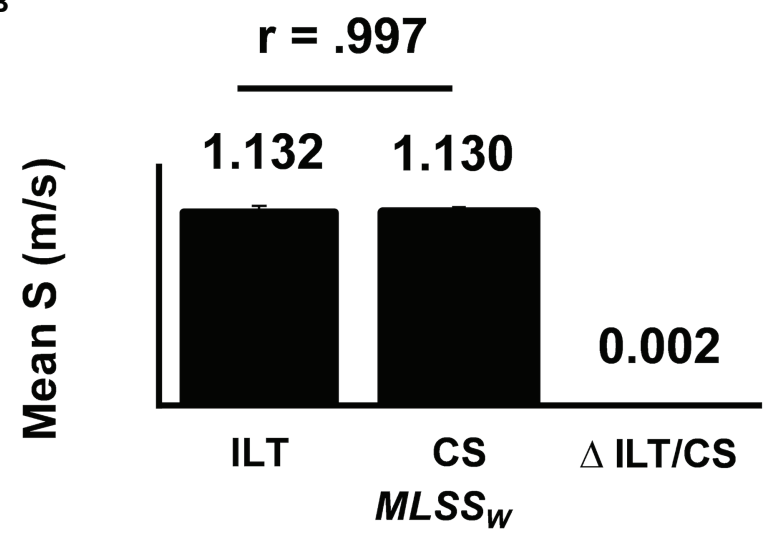

FIGURE 6 | Lactate dynamics at the individual lactate threshold (ILT). (A) Relationship between workload $(\mathrm{WL})$ variation $(\Delta S)$ and blood lactate dynamics $\left(\Delta\left[\mathrm{La}^{-}\right]\right)$at the ILT using threshold criterion 1 and 2 (TC1/2). A rapid blood lactate accumulation $(>0.4 \mathrm{Mm})$ occurs in response to a minute $\mathrm{WL}$ increase $(0.01 \mathrm{~m} / \mathrm{s})$ after $1-6$ steps at constant speed. While increments of $0.015 \mathrm{~m} / \mathrm{s}$ and $0.025 \mathrm{~m} / \mathrm{s}$ do not differ from $0.01 \mathrm{~m} / \mathrm{s}$, a step of $0.05 \mathrm{~m} / \mathrm{s}$ provokes another significant lactate accumulation $(0.05 \mathrm{~m} / \mathrm{s}$ vs. $0.025 \mathrm{~m} / \mathrm{s}$; $0.05 \mathrm{~m} / \mathrm{s}$ vs. $0.015 \mathrm{~m} / \mathrm{s}, 0.05 \mathrm{~m} / \mathrm{s}$ vs. $0.01 \mathrm{~m} / \mathrm{s}, N=65$, ${ }^{\star * \star} p<0.001$, twotailed unpaired $t$-tests). (B) Reliability and validity of the ILT-test. Intraindividual day to day comparison of the maximal lactate steady state workload (MLSS ${ }_{w}$ ) obtained by an ILT-test and a subsequent constant speed (CS)-test (ILTtest $=1.132 \mathrm{~m} / \mathrm{s}$, CS-test $=1.130 \mathrm{~m} / \mathrm{s}, \Delta$ ILT-test $/$ CS-test $=0.002 \mathrm{~m} / \mathrm{s}$, $\mathrm{CV}=0.49 \%, n=13, r=0.997, p<0.001$ ). Data are mean $\pm \mathrm{SE}$. [La-], arterial lactate concentration; $\mathrm{S}$, speed.

quality, and exercise intensity to prime neuromuscular plasticity. Given the complexity of such highly individual and movementspecific processes, attention should be paid toward application of reliable, valid, and unbiased reference parameters, which capture exercise intensities in the form of speed (running and swimming) or power (cycling). There is compelling evidence that the $\mathrm{MLSS}_{\mathrm{W}}$ is the most appropriate parameter to meet these requirements (Jamnick et al., 2020; for excellent reviews see also (Fluck and Hoppeler, 2003; Bishop et al., 2019). Although the $\mathrm{MLSS}_{\mathrm{W}}$ has repeatedly been described as a reliable marker and accurate predictor of subthreshold endurance capacity in running and cycling (Heck et al., 1985; Hering et al., 2018; Jamnick et al., 2018), its relevance for suprathreshold intensities of exercise is unknown. Here, we have adapted a recently introduced single-visit test (ILT-test) for reliable determination of the $\mathrm{MLSS}_{\mathrm{W}}$ in running and cycling for swimming and showed that swimming speed in competitions up to $1,500 \mathrm{~m}$ is well above the MLSS $_{\mathrm{W}}$. Moreover, absolute $\mathrm{MLSS}_{\mathrm{W}}$ level and the difference between the $\mathrm{MLSS}_{\mathrm{W}}$ and swimming speed in competition correlate well with general swimming performance, in particular with supra-threshold endurance capacity. Importantly, a training-related combination of a high $\mathrm{MLSS}_{\mathrm{W}}$ and a high $\Delta \mathrm{S}$-Comp/MLSS $\mathrm{W}_{\mathrm{W}}$ is more likely to be found in long-distance trained athletes than in shortdistance trained athletes. Consequently, these data suggest that the $\mathrm{MLSS}_{\mathrm{W}}$ is a reliable submaximal parameter for predicting endurance capacity below and above the LT and to set the optimal training intensity even in severe domains of exercise.

We recently provided a detailed discussion on physiological meanings of the ILT-test (Hering et al., 2018). Two issues are of particular interest and illustrate the relevance of the test procedure to exercise physiology: first, the extremely fine-tuned regulation of lactate homeostasis, which can be captured by the ILT-test. Minute workload variations while running, cycling, and swimming at the MLSS $_{\mathrm{W}}(0.1 \mathrm{~m} / \mathrm{s} / 7 \mathrm{~W} / 0.01 \mathrm{~m} / \mathrm{s})$ generate drastic changes in blood lactate levels (Figure 6A). Second, ILT-test results are highly reproducible. The test-retest coefficients for running and swimming are 0.996 and 0.997, respectively (Hering et al., 2018).

We previously demonstrated a strong correlation between the MLSS $_{\mathrm{W}}$ and performance in half-marathon competition ( $r=0.96$; Hering et al., 2018). This allows a remarkably precise prediction of race results (Hering et al., 2018). Compared to half-marathon runners, we found a lower correlation between the $\mathrm{MLSS}_{\mathrm{W}}$ and the average swimming pace in competition (S-Comp, 0.96 vs. 0.87). Hence, S-Comp in swimmers was on average $3.4 \%$ above the MLSS $_{\mathrm{W}}$ (data not shown), while halfmarathon runners exercised 5.2\% below (Hering et al., 2018). Moreover, we found differences in swimming speed (S-Comp-L vs. S-Comp-SM) and for the gap between $\mathrm{MLSS}_{\mathrm{W}}$ and S-Comp $\left(\Delta\right.$ S-Comp-L/MLSS ${ }_{\mathrm{W}}$-L vs. $\Delta$ S-Comp-SM/MLSS ${ }_{\mathrm{W}}-\mathrm{SM}$ ) when comparing long-distance (L, 400-1,500 m), and short-/middledistance $(50-200 \mathrm{~m})$ athletes (SM; Figure 7B). The $\mathrm{MLSS}_{\mathrm{W}} \mathrm{did}$ not differ between the two groups (Figure 7B). Therefore, in contrast to half-marathon running, race results in swimming cannot be explained by the $\mathrm{MLSS}_{\mathrm{W}}$ level alone. Rather, these data provide substantial evidence that the WL range above the $\mathrm{MLSS}_{\mathrm{W}}$ is a major factor in swimming and probably in sports with comparable racing time. Thus, we speculate, that both, a high MLSS $_{\mathrm{W}}$ and a large $\Delta \mathrm{S}$-Comp/MLSS $\mathrm{W}$ are crucial in Olympic long-distance swimming (Figures 5, 7A,C,D). Finally, we found a stronger correlation $(r=0.9)$ between $\mathrm{MLSS}_{\mathrm{W}}-\mathrm{L}$ and S-Comp-L compared to MLSS $_{\mathrm{W}}$-SM and S-Comp-SM (0.84; Figure 7B), while only the correlation between $\Delta \mathrm{MLSS}_{\mathrm{W}}-\mathrm{L} / \mathrm{S}$ Comp-L vs. S-Comp-L was significant ( $p<0.05$; data not shown). In long-distance swimmers, this indicates an increased fatigue resistance of the muscle fibers that are recruited above the MLSS $S_{\mathrm{W}}$. 

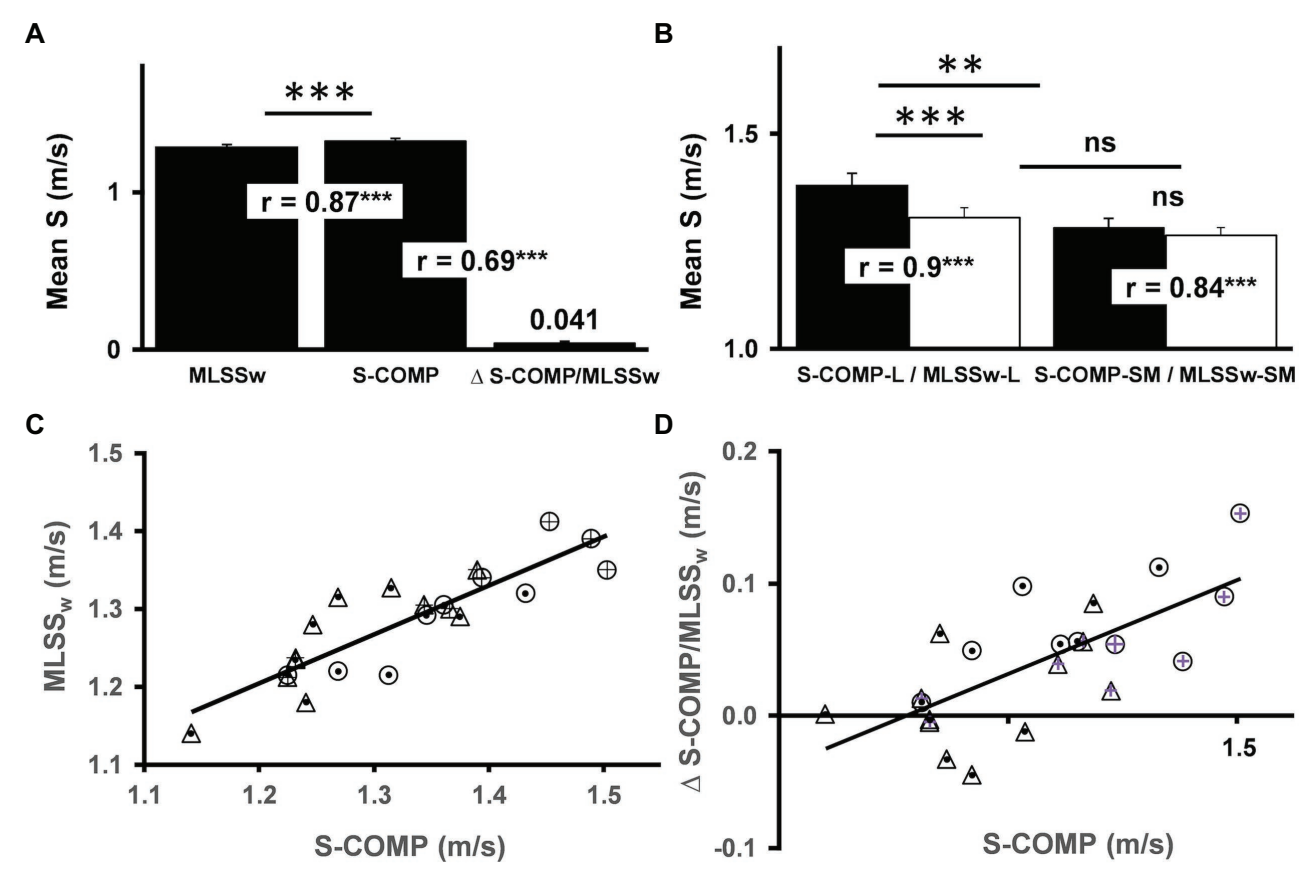

FIGURE 7 | Comparison of test and race results. (A) Comparison of swimming speed at MLSS ${ }_{\mathrm{w}}$ and swimming speed in competition (S-Comp) and their difference ( $\Delta$ S-Comp/MLSS ${ }_{w}$ : MLSS $: 1.283 \mathrm{~m} / \mathrm{s}$ vs. S-Comp: 1.326, ${ }^{* * * *} p<0.001$, two-tailed paired $t$-test, $r=0.87, * * * 0<0.001$; S-Comp: $1.326 \mathrm{~m} / \mathrm{s}$ vs. $\Delta S-C o m p / M L S S_{w}:$ $\left.0.041 \mathrm{~m} / \mathrm{s}, r=0.69,{ }^{* * * *} p<0.001 ; n=22\right)$. (B) Comparison of mean swimming speed at $\mathrm{MLSS}_{\mathrm{w}}$ and in competition (800 and 1,500 m, S-Comp) between longdistance swimmers (L, $n=10$ ) and middle- and short-distance swimmers (SM, $n=12$; S-Comp-L: 1.378 vs. MLSS $w$-L: 1.305 , ${ }^{* * * *} p<0.001$, two-tailed paired $t$-test, $r=0.9,{ }^{* * *} p<0.001$; S-Comp-SM: 1.281 vs. MLSS ${ }_{w}-S M: 1.264$, ns., two-tailed paired $t$-test, $r=0.84,{ }^{* * * *} p<0.001$; S-Comp-L: $1.378 \mathrm{~m} / \mathrm{s}$ vs. S-Comp-SM:

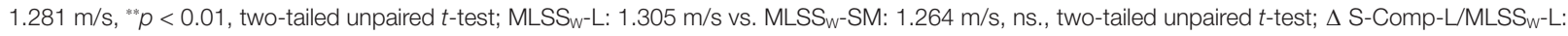
$0.072 \mathrm{~m} / \mathrm{s}$ vs. $\Delta$ S-Comp-SM/MLSS ${ }_{w}-S M: 0.015 \mathrm{~m} / \mathrm{s}^{, *} p<0.01$, two-tailed unpaired $t$-test). (C) Relationship between swimming speed at the MLSS ${ }_{w}$ and swimming speed in competition ( $r=0.87, p<0.001 ; \mathrm{O}=$ long distance group (L), $\Delta=$ middle and short distance group (SM), $+=$ men, $\bullet=$ women).

(D) Relationship between $\Delta$ S-Comp/MLSS $w$ and S-Comp $(r=0.698, p<0.001)$. Data are mean \pm SE. S, speed.

With increasing WL demands at the MLSS ${ }_{\mathrm{W}}$, fast-twitching and more glycolytic type II fibers get more likely recruited (Henneman et al., 1965; Burke et al., 1971; Peter et al., 1972; Vollestad and Blom, 1985). The accompanying blood lactate accumulation will rapidly exceed clearance systems and is commonly referred to as LT. Exercise in this intensity range can trigger the following adaptions: (i) higher mitochondrial content and/or activity (Dudley et al., 1982; Howald et al., 1985; Hoppeler, 1986; Daussin et al., 2008), increased capillarization (Li et al., 2020), (ii) higher MCT1/MCT4 content (Dubouchaud et al., 2000; de Araujo et al., 2015) and (iii) an overall increase in lactate clearance rate (Bergman et al., 1999; Messonnier et al., 2013; for comprehensive reviews, see Fluck and Hoppeler, 2003; Schiaffino and Reggiani, 2011; Hoppeler, 2016). These adaptions may at least partially explain the higher fatigue resistance above the $\mathrm{MLSS}_{\mathrm{W}}$ of long-distance swimmers (800-1,500 m).

Fast-twitching muscle fibers consume considerably more ATP and $\mathrm{O}_{2}$ (Greenhaff et al., 1993; Stienen et al., 1995; He et al., 2000; Szentesi et al., 2001). Converting them into muscles with a higher aerobic capacity to expand supra-threshold endurance capacity is challenging in humans, since everyday movements counteract eventual training interventions (Monster et al., 1978; Lindstedt et al., 1991; Pette and Staron, 1997). A conversion of muscle fibers from MHC IIa type to MHC I type, with the same or larger fiber diameter has been demonstrated in cyclists (Coyle et al., 1992; Horowitz et al., 1994; Coyle, 2005) and in a bicycle ergometer training-study at $75 \%$ peak oxygen consumption (Dubouchaud et al., 2000). Importantly, the plasticity of muscles was correlated with higher endurance capacity (Coyle et al., 1992; Horowitz et al., 1994; Coyle, 2005) possibly because the ATP consumption of type I fibers is comparatively lower for the same force production (Greenhaff et al., 1993; Stienen et al., 1996; He et al., 2000; Szentesi et al., 2001). However, there would only be a net advantage if the discipline-specific movement execution corresponds to the twitching properties of performance-relevant muscles/muscle fibers (Tesch and Karlsson, 1985). To our knowledge, no bioptically proven, training-induced fiber transformation in swimming has been reported so far. Comparison of highly-trained elite $1,500 \mathrm{~m}$ swimmers and amateurs demonstrated larger and more type I fibers in the M. vastus lateralis of elite swimmers (Gerard et al., 1986). Although exercise induced fiber transformation from fast to slow after several years of training is a possible explanation for the higher S-Comp of long-distance swimmers, data on arm muscles is not available.

Recent studies show that training stimuli in the MLSS $_{\mathrm{w}}$ range (Dubouchaud et al., 2000) induce not only muscle fiber 
transformation but also prime neuronal plasticity in severe domains of exercise (Pin-Barre et al., 2017; Rahmati and Kazemi, 2019; Constans et al., 2021). Complicating this picture, metabolic homeostasis in swimming is more dependent on technical skills than in running or cycling (Figueiredo et al., 2014). For example, elite swimmers use different combinations of stroke parameters compared to amateur-level athletes (Seifert et al., 2010b). Moreover, several studies consistently found increased stroke frequency accompanied by decreased stroke length slightly above the MLSS ${ }_{\mathrm{W}}$ (Oliveira et al., 2012; Figueiredo et al., 2013, 2014; Pelarigo et al., 2017). Alberty et al. assume that a change in arm coordination has two key benefits: (i) a better chain of the propulsive actions and (ii) a greater time allotted to propulsion per distance unit during increasing muscular fatigue (Alberty et al., 2009; Seifert et al., 2010a).

A study on open water swimmers (OWSs, 5-25 km) and short distance swimmers (SDSs, 50-100 m), two subgroups comparable to the present study, clearly demonstrates the strong relationship between muscle coordination and energy metabolism (Seifert et al., 2010a). In an intensity-adjusted progressive $6 \times 300 \mathrm{~m}$ test, the OWS achieved a higher mean speed (1.39 vs. $\left.1.27 \mathrm{~m} \cdot \mathrm{s}^{-1}\right)$, stroke rate $(0.62$ vs. $0.55 \mathrm{~Hz})$, and stroke index $\left(\mathrm{SI}=\mathrm{SL} \cdot \mathrm{SV}, 3.19\right.$ vs. $\left.2.96 \mathrm{~m}^{2} \mathrm{~s}^{-1}\right)$, and a lower stroke length (SL, 2.28 vs. $2.31 \mathrm{~m} \cdot \mathrm{stroke}^{-1}$ ) and index of coordination (IdC, -21.7 vs. $-11.2 \%)$ than the SDS. When a lag time occurred between the propulsive phases of the two arms, the stroke coordination was called as "catch-up" (IdC $<0 \%$; Chollet et al., 2000). Importantly, the OWS, who should be highly fatigue resistant, kept their coordination in great catch-up, presumably because they were highly focused on their hydro-dynamic position in order to minimize resistive forces and maximize efficiency (Seifert et al., 2010a). The relationship between increasing energy consumption and decreasing catch-up phase was observed in both groups [IdC vs. energy cost $(C), p<0.05]$, but the OWS had longer glide phases in catch-up mode (IdC) and higher stroke efficiency (SI) for the same energy consumption, resulting in more efficient propulsion. Moreover, on the average of six progressive $300 \mathrm{~m}$ sets beginning with a swimming speed near the MLSS $_{\mathrm{W}}(0.1 \mathrm{~m} / \mathrm{s}$ below the $400 \mathrm{~m}$ best time, increment $=0.017 \mathrm{~m} / \mathrm{s}$ ), the OWS also had lower blood lactate concentrations ( 3.1 vs. $5.9 \mathrm{mmol} \cdot \mathrm{L}^{-1}$ ), lower energy consumption (C, 12.89 vs. $\left.14.8 \mathrm{~J} \cdot \mathrm{kg}^{-1} \cdot \mathrm{m}^{-1}\right)$, and a greater net $\mathrm{VO}_{2}(2,903$ vs. $2,825 \mathrm{ml} \cdot \mathrm{min}^{-1}$ ) than the SDS (Seifert et al., 2010a). This is in line with our data showing a higher S-Comp and a larger $\Delta$ S-Comp/MLSS $S_{\mathrm{W}}$ in $800-1,500 \mathrm{~m}$ swimmers. In accordance with Seifert et al. (2010a), we hypothesize that long-distance trained swimmers, and likely endurance athletes with comparable racing times, have a higher fatigue resistance in the supra-threshold range due to improvements in technique and metabolism. Training intensity/duration adjustment in moderate to severe domains of exercise will trigger neuromuscular adaptations (Dudley et al., 1982; Dubouchaud et al., 2000; Messonnier et al., 2013; Figueiredo et al., 2014; de Araujo et al., 2015), which most likely manifest in a higher MLSS $_{W}$ (Billat et al., 2004; Philp et al., 2008). Such a higher fatigue resistance in the supra-threshold range can facilitate economic movement over longer periods of time.
Together with the improved coordination patterns described above, peripheral and/or central recruitment of additional motor units and their higher discharge frequencies can be converted into strength increases (Henneman et al., 1965; Sale, 1988; Wakeling et al., 2002, 2006, 2012; Jensen et al., 2005; Ansdell et al., 2020). This ultimately leads to higher muscle power $($ Power $=$ Force $\cdot$ Velocity/Speed $)$ with a constant fiber/muscle cross-section and thus also a constant weight (Gabriel et al., 2006). A further increase in strength can be achieved by increasing the cross-sectional area of muscles through unspecific or specific strength training (Fyfe et al., 2014). However, it is important to note, that the isolated stimulation of muscle hypertrophy neglects neuromuscular optimization, which limits its application in competitive sports. Nevertheless, an elevated fiber/muscle cross-section will likely result in improved performance if $\Delta \mathrm{MLSS}_{\mathrm{W}} / \mathrm{S}$-Comp increases at low or constant MLSS $_{\mathrm{w}}$. Such a scenario may also explain the performance profile of TP4 (Figure 5, see also the next paragraph).

An in-depth analysis of complete sets of Olympic distances (Figure 5) reveals significant differences in $\mathrm{MLSS}_{\mathrm{W}}$ scores and for the gap between MLSS $_{\mathrm{W}}$ and swimming pace in competition $\left(\Delta\right.$ S-Comp/MLSS ${ }_{\mathrm{W}}$ ). The comparatively high $\mathrm{MLSS}_{\mathrm{W}}$ of TP1 indicates substantial neuromuscular adaptations, which may explain the race results above the average in all disciplines. Other athletes (TP2-4) seem to have less well coordinated movement patterns, likely underlying their lower threshold speeds $\left(\mathrm{MLSS}_{\mathrm{W}}\right)$. TP4 has the fastest $50 \mathrm{~m}$ time, but a considerably lower MLSS $_{\mathrm{W}}$ manifesting in a rapid decline in performance starting from $200 \mathrm{~m}$. Although at a lower level, $\Delta$ S-Comp/ MLSS $_{\mathrm{W}}$ of TP2 follows the course of TP1 indicating a similar history of training stimuli. In contrast, the relatively high $\Delta$ S-Comp/MLSS ${ }_{\mathrm{W}}$ for distances up to $400 \mathrm{~m}$ and the decline in speed at 800 and $1,500 \mathrm{~m}$ of TP4 suggests insufficient aerobic neuromuscular adaptation of muscle fibers involved in supra-threshold exercise.

\section{CONCLUSION}

Considerable progress has been made in understanding the molecular and neuromuscular architecture of adaptation processes to various training stimuli (Fluck and Hoppeler, 2003; Fyfe et al., 2014; Granata et al., 2018; Bishop et al., 2019). However, successful translation of basic insights into useful training concepts has been constrained by the lack of appropriate reference parameters (Jamnick et al., 2020). Using a sophisticated single-exercise test in swimming and results from swimming competitions, we provide clear evidence that the MLSS $_{\mathrm{W}}$ is a reliable and valid parameter to assess endurance capacity in moderate to severe domains of exercise (Hering et al., 2018; Jamnick et al., 2020). Consequently, we suggest that personal training recommendations and monitoring of exercise-induced changes in individual performance level should be calculated relative to the $\mathrm{MLSS}_{\mathrm{w}}$ rather than to peak or maximal workload parameters (Baldwin et al., 2000; Skorski et al., 2012; Bishop et al., 2019). 
DATA AVAILABILITY STATEMENT

The raw data supporting the conclusions of this article will be made available by the authors, without undue reservation.

\section{ETHICS STATEMENT}

The studies involving human participants were reviewed and approved by the Ethics Committee (institutional review board, IRB) of the University of Konstanz. Written informed consent to participate in this study was provided by the participants' legal guardian/next of kin.

\section{AUTHOR CONTRIBUTIONS}

GH developed the hardware and software, conceived and designed research, performed experiments, analyzed data, prepared and interpreted results, drafted the manuscript, prepared

\section{REFERENCES}

Alberty, M., Sidney, M., Pelayo, P., and Toussaint, H. M. (2009). Stroking characteristics during time to exhaustion tests. Med. Sci. Sports Exerc. 41, 637-644. doi: 10.1249/MSS.0b013e31818acfba

Ansdell, P., Brownstein, C. G., Skarabot, J., Angius, L., Kidgell, D., Frazer, A., et al. (2020). Task-specific strength increases after lower-limb compound resistance training occurred in the absence of corticospinal changes in vastus lateralis. Exp. Physiol. 105, 1132-1150. doi: 10.1113/ EP088629

Baldari, C., Bonavolonta, V., Emerenziani, G. P., Gallotta, M. C., Silva, A. J., and Guidetti, L. (2009). Accuracy, reliability, linearity of Accutrend and Lactate Pro versus EBIO plus analyzer. Eur. J. Appl. Physiol. 107, 105-111. doi: 10.1007/s00421-009-1107-5

Baldwin, J., Snow, R. J., and Febbraio, M. A. (2000). Effect of training status and relative exercise intensity on physiological responses in men. Med. Sci. Sports Exerc. 32, 1648-1654. doi: 10.1097/00005768200009000-00020

Batschelet, A. (2004). Reproduzierbarkeit des maximalen Laktat-steady-states. SEMS 52, 154-156.

Bentley, D. J., Newell, J., and Bishop, D. (2007). Incremental exercise test design and analysis: implications for performance diagnostics in endurance athletes. Sports Med. 37, 575-586. doi: 10.2165/00007256200737070-00002

Bergman, B. C., Wolfel, E. E., Butterfield, G. E., Lopaschuk, G. D., Casazza, G. A., Horning, M. A., et al. (1999). Active muscle and whole body lactate kinetics after endurance training in men. J. Appl. Physiol. 87, 1684-1696. doi: 10.1152/ jappl.1999.87.5.1684

Billat, V., Sirvent, P., Lepretre, P. M., and Koralsztein, J. P. (2004). Training effect on performance, substrate balance and blood lactate concentration at maximal lactate steady state in master endurance-runners. Pflugers Arch. 447, 875-883. doi: 10.1007/s00424-003-1215-8

Billat, V. L., Sirvent, P., Py, G., Koralsztein, J. P., and Mercier, J. (2003). The concept of maximal lactate steady state: a bridge between biochemistry, physiology and sport science. Sports Med. 33, 407-426. doi: 10.2165/ 00007256-200333060-00003

Bishop, D. J., Botella, J., Genders, A. J., Lee, M. J., Saner, N. J., Kuang, J., et al. (2019). High-intensity exercise and mitochondrial biogenesis: current controversies and future research directions. Physiology 34, 56-70. doi: 10.1152/physiol.00038.2018

Borg, G. A. (1982). Psychophysical bases of perceived exertion. Med. Sci. Sports Exerc. 14, 377-381. figures, and edited and revised the manuscript. JS performed experiments, interpreted results, prepared figures, and edited and revised the manuscript.

\section{ACKNOWLEDGMENTS}

We sincerely thank the test persons and the BA and MA students, namely Patrick Thumm, Mike Baumann, Benjamin Singer, Christian Bächle, Frederic Wortmeier, Patricia Scheller, and Jasmin Wagner for their dedication and participation in this study. We also gratefully acknowledge the technical support of the engineering department, namely Harald Kautz, Hubert Fink, Harald Richter, and Bruno Erne. Furthermore, we would like to thank the representatives of the sports department of the city of Konstanz, especially Roland Lohr for the opportunity to generously use the municipal swimming pool. Finally, we would like to thank the Research Committee of the University of Konstanz for financial support.

Brooks, G. A. (1985a). Anaerobic threshold: review of the concept and directions for future research. Med. Sci. Sports Exerc. 17, 22-34.

Brooks, G. A. (1985b). "Lactate:Glycolytic End Product and Oxidative Substrate During Sustained Exercise in Mammals - the "Lactate Shuttle,"' in Circulation, Respiration, and Metabolism: Current Comparative Approaches. ed. R. Gilles (Berlin; New York: Springer-Verlag).

Burke, R. E., Levine, D. N., and Zajac, F. E. 3rd. (1971). Mammalian motor units: physiological-histochemical correlation in three types in cat gastrocnemius. Science 174, 709-712. doi: 10.1126/science.174.4010.709

Chollet, D., Chalies, S., and Chatard, J. C. (2000). A new index of coordination for the crawl: description and usefulness. Int. J. Sports Med. 21, 54-59. doi: 10.1055/s-2000-8855

Constans, A., Pin-Barre, C., Molinari, F., Temprado, J. J., Brioche, T., Pellegrino, C., et al. (2021). High-intensity interval training is superior to moderate intensity training on aerobic capacity in rats: impact on hippocampal plasticity markers. Behav. Brain Res. 398:112977. doi: 10.1016/j.bbr.2020.112977

Coyle, E. F. (2005). Improved muscular efficiency displayed as Tour de France champion matures. J. Appl. Physiol. 98, 2191-2196. doi: 10.1152/ japplphysiol.00216.2005

Coyle, E. F., Sidossis, L. S., Horowitz, J. F., and Beltz, J. D. (1992). Cycling efficiency is related to the percentage of type I muscle fibers. Med. Sci. Sports Exerc. 24, 782-788.

Dassonville, J., Beillot, J., Lessard, Y., Jan, J., Andre, A. M., Le Pourcelet, C., et al. (1998). Blood lactate concentrations during exercise: effect of sampling site and exercise mode. J. Sports Med. Phys. Fitness 38, 39-46.

Daussin, F. N., Zoll, J., Dufour, S. P., Ponsot, E., Lonsdorfer-Wolf, E., Doutreleau, S., et al. (2008). Effect of interval versus continuous training on cardiorespiratory and mitochondrial functions: relationship to aerobic performance improvements in sedentary subjects. Am. J. Physiol. Regul. Integr. Comp. Physiol. 295, R264-R272. doi: 10.1152/ajpregu.00875.2007

de Araujo, G. G., Gobatto, C. A., de Barros Manchado-Gobatto, F., Teixeira, L. F., Dos Reis, I. G., Caperuto, L. C., et al. (2015). MCT1 and MCT4 kinetic of mRNA expression in different tissues after aerobic exercise at maximal lactate steady state workload. Physiol. Res. 64, 513-522. doi: 10.33549/ physiolres. 932695

Donovan, C. M., and Brooks, G. A. (1983). Endurance training affects lactate clearance, not lactate production. Am. J. Phys. 244, E83-E92. doi: 10.1152/ ajpendo.1983.244.1.E83

Dubouchaud, H., Butterfield, G. E., Wolfel, E. E., Bergman, B. C., and Brooks, G. A. (2000). Endurance training, expression, and physiology of LDH, MCT1, and MCT4 in human skeletal muscle. Am. J. Physiol. Endocrinol. Metab. 278, E571-E579. doi: 10.1152/ajpendo.2000.278.4.E571 
Dudley, G. A., Abraham, W. M., and Terjung, R. L. (1982). Influence of exercise intensity and duration on biochemical adaptations in skeletal muscle. $J$. Appl. Physiol. Respir. Environ. Exerc. Physiol. 53, 844-850. doi: 10.1152/ jappl.1982.53.4.844

Faude, O., Kindermann, W., and Meyer, T. (2009). Lactate threshold concepts: how valid are they? Sports Med. 39, 469-490. doi: 10.2165/ 00007256-200939060-00003

Feliu, J., Ventura, J. L., Segura, R., Rodas, G., Riera, J., Estruch, A., et al. (1999). Differences between lactate concentration of samples from ear lobe and the finger tip. J. Physiol. Biochem. 55, 333-339.

Figueiredo, P., Morais, P., Vilas-Boas, J. P., and Fernandes, R. J. (2013). Changes in arm coordination and stroke parameters on transition through the lactate threshold. Eur. J. Appl. Physiol. 113, 1957-1964. doi: 10.1007/s00421-013-2617-8

Figueiredo, P., Nazario, R., Sousa, M., Pelarigo, J. G., Vilas-Boas, J. P., and Fernandes, R. (2014). Kinematical analysis along maximal lactate steady state swimming intensity. J. Sports Sci. Med. 13, 610-615.

FINA, F.I.d.N. (2021). Overall rankings world records swimming men. Available at: http://www.fina.org/fina-rankings/results? recordCode=WR\&gender=Men\& poolConfiguration $=$ LCM\&type $=$ records\&selectedGroup $=$ Freestyle $\&$ selected $G$ ender $=$ Men\&selectedDistance $=50$ (Accessed January 21, 2021).

Fluck, M., and Hoppeler, H. (2003). Molecular basis of skeletal muscle plasticityfrom gene to form and function. Rev. Physiol. Biochem. Pharmacol. 146, 159-216. doi: 10.1007/s10254-002-0004-7

Fyfe, J. J., Bishop, D. J., and Stepto, N. K. (2014). Interference between concurrent resistance and endurance exercise: molecular bases and the role of individual training variables. Sports Med. 44, 743-762. doi: 10.1007/ s40279-014-0162-1

Gabriel, D. A., Kamen, G., and Frost, G. (2006). Neural adaptations to resistive exercise: mechanisms and recommendations for training practices. Sports Med. 36, 133-149. doi: 10.2165/00007256-200636020-00004

Gerard, E. S., Caiozzo, V. J., Rubin, B. D., Prietto, C. A., and Davidson, D. M. (1986). Skeletal muscle profiles among elite long, middle, and short distance swimmers. Am. J. Sports Med. 14, 77-82. doi: 10.1177/036354658601400113

Granata, C., Jamnick, N. A., and Bishop, D. J. (2018). Training-induced changes in mitochondrial content and respiratory function in human skeletal muscle. Sports Med. 48, 1809-1828. doi: 10.1007/s40279-018-0936-y

Greenhaff, P. L., Soderlund, K., Ren, J. M., and Hultman, E. (1993). Energy metabolism in single human muscle fibres during intermittent contraction with occluded circulation. J. Physiol. 460, 443-453. doi: 10.1113/jphysiol.1993. sp019480

Hauser, T., Bartsch, D., Baumgartel, L., and Schulz, H. (2013). Reliability of maximal lactate-steady-state. Int. J. Sports Med. 34, 196-199. doi: 10.1055/ s-0032-1321719

He, Z. H., Bottinelli, R., Pellegrino, M. A., Ferenczi, M. A., and Reggiani, C. (2000). ATP consumption and efficiency of human single muscle fibers with different myosin isoform composition. Biophys. J. 79, 945-961. doi: 10.1016/S0006-3495(00)76349-1

Heck, H., Mader, A., Hess, G., Mucke, S., Muller, R., and Hollmann, W. (1985). Justification of the 4-mmol/l lactate threshold. Int. J. Sports Med. 6, 117-130. doi: $10.1055 / \mathrm{s}-2008-1025824$

Henneman, E., Somjen, G., and Carpenter, D. O. (1965). Excitability and inhibitability of motoneurons of different sizes. J. Neurophysiol. 28, 599-620. doi: $10.1152 /$ jn.1965.28.3.599

Hering, G. O., Hennig, E. M., Riehle, H. J., and Stepan, J. (2018). A lactate kinetics method for assessing the maximal lactate steady state workload. Front. Physiol. 9:310. doi: 10.3389/fphys.2018.00310

Hoppeler, H. (1986). Exercise-induced ultrastructural changes in skeletal muscle. Int. J. Sports Med. 7, 187-204. doi: 10.1055/s-2008-1025758

Hoppeler, H. (2016). Molecular networks in skeletal muscle plasticity. J. Exp. Biol. 219, 205-213. doi: 10.1242/jeb.128207

Horowitz, J. F., Sidossis, L. S., and Coyle, E. F. (1994). High efficiency of type I muscle fibers improves performance. Int. J. Sports Med. 15, 152-157. doi: 10.1055/s-2007-1021038

Howald, H., Hoppeler, H., Claassen, H., Mathieu, O., and Straub, R. (1985). Influences of endurance training on the ultrastructural composition of the different muscle fiber types in humans. Pflugers Arch. 403, 369-376. doi: 10.1007/BF00589248

Jamnick, N. A., Botella, J., Pyne, D. B., and Bishop, D. J. (2018). Manipulating graded exercise test variables affects the validity of the lactate threshold and [Formula: see text]. PLoS One 13:e0199794. doi: 10.1371/journal. pone.0199794

Jamnick, N. A., Pettitt, R. W., Granata, C., Pyne, D. B., and Bishop, D. J. (2020). An examination and critique of current methods to determine exercise intensity. Sports Med. 50, 1729-1756. doi: 10.1007/s40279-020-01322-8

Jensen, J. L., Marstrand, P. C., and Nielsen, J. B. (2005). Motor skill training and strength training are associated with different plastic changes in the central nervous system. J. Appl. Physiol. 99, 1558-1568. doi: 10.1152/ japplphysiol.01408.2004

Li, J., Li, Y., Atakan, M. M., Kuang, J., Hu, Y., Bishop, D. J., et al. (2020). The molecular adaptive responses of skeletal muscle to high-intensity exercise/ training and hypoxia. Antioxidants 9:656. doi: 10.3390/antiox9080656

Lindstedt, S. L., Hokanson, J. F., Wells, D. J., Swain, S. D., Hoppeler, H., and Navarro, V. (1991). Running energetics in the pronghorn antelope. Nature 353, 748-750. doi: $10.1038 / 353748 \mathrm{a} 0$

Mamen, A. (2014). "Lactate Pro vs Lactate Pro2," in 19th annual Congress of the European College of Sport Science. eds. A. De Haan, C. J. De Ruiter, and E. Tsolakidis (Amsterdam VU University Amsterdam \& VU University Medical Center).

Messonnier, L. A., Emhoff, C. A., Fattor, J. A., Horning, M. A., Carlson, T. J., and Brooks, G. A. (2013). Lactate kinetics at the lactate threshold in trained and untrained men. J. Appl. Physiol. 114, 1593-1602. doi: 10.1152/ japplphysiol.00043.2013

Monster, A. W., Chan, H., and O'Connor, D. (1978). Activity patterns of human skeletal muscles: relation to muscle fiber type composition. Science 200, 314-317. doi: $10.1126 /$ science.635587

Oliveira, M. F., Caputo, F., Dekerle, J., Denadai, B. S., and Greco, C. C. (2012). Stroking parameters during continuous and intermittent exercise in regionallevel competitive swimmers. Int. J. Sports Med. 33, 696-701. doi: 10.1055/ s-0031-1298003

Pelarigo, J. G., Machado, L., Fernandes, R. J., Greco, C. C., and Vilas-Boas, J. P. (2017). Oxygen uptake kinetics and energy system's contribution around maximal lactate steady state swimming intensity. PLoS One 12:e0167263. doi: 10.1371/journal.pone.0167263

Peter, J. B., Barnard, R. J., Edgerton, V. R., Gillespie, C. A., and Stempel, K. E. (1972). Metabolic profiles of three fiber types of skeletal muscle in Guinea pigs and rabbits. Biochemistry 11, 2627-2633. doi: 10.1021/bi00764a013

Pette, D., and Staron, R. S. (1997). Mammalian skeletal muscle fiber type transitions. Int. Rev. Cytol. 170, 143-223. doi: 10.1016/s0074-7696(08)61622-8

Philp, A., Macdonald, A. L., Carter, H., Watt, P. W., and Pringle, J. S. (2008). Maximal lactate steady state as a training stimulus. Int. J. Sports Med. 29, 475-479. doi: 10.1055/s-2007-965320

Pin-Barre, C., Constans, A., Brisswalter, J., Pellegrino, C., and Laurin, J. (2017). Effects of high- versus moderate-intensity training on neuroplasticity and functional recovery after focal ischemia. Stroke 48, 2855-2864. doi: 10.1161/ STROKEAHA.117.017962

Pyne, D. B., Boston, T., Martin, D. T., and Logan, A. (2000). Evaluation of the Lactate Pro blood lactate analyser. Eur. J. Appl. Physiol. 82, 112-116. doi: $10.1007 / \mathrm{s} 004210050659$

Pyne, D. B., Lee, H., and Swanwick, K. M. (2001). Monitoring the lactate threshold in world-ranked swimmers. Med. Sci. Sports Exerc. 33, 291-297. doi: 10.1097/00005768-200102000-00019

Rahmati, M., and Kazemi, A. (2019). Various exercise intensities differentially regulate GAP-43 and CAP-1 expression in the rat hippocampus. Gene 692 185-194. doi: 10.1016/j.gene.2019.01.013

Sale, D. G. (1988). Neural adaptation to resistance training. Med. Sci. Sports Exerc. 20 (Suppl. 5), S135-S145. doi: 10.1249/00005768-198810001-00009

Schiaffino, S., and Reggiani, C. (2011). Fiber types in mammalian skeletal muscles. Physiol. Rev. 91, 1447-1531. doi: 10.1152/physrev.00031.2010

Seifert, L., Komar, J., Lepretre, P. M., Lemaitre, F., Chavallard, F., Alberty, M., et al. (2010a). Swim specialty affects energy cost and motor organization. Int. J. Sports Med. 31, 624-630. doi: 10.1055/s-0030-1255066

Seifert, L., Toussaint, H. M., Alberty, M., Schnitzler, C., and Chollet, D. (2010b). Arm coordination, power, and swim efficiency in national and regional front crawl swimmers. Hum. Mov. Sci. 29, 426-439. doi: 10.1016/j.humov.2009.11.003

Skorski, S., Faude, O., Urhausen, A., Kindermann, W., and Meyer, T. (2012). Intensity control in swim training by means of the individual anaerobic threshold. J. Strength Cond. Res. 26, 3304-3311. doi: 10.1519/ JSC.0b013e31824b6014 
Stainsby, W. N., and Brooks, G. A. (1990). Control of lactic acid metabolism in contracting muscles and during exercise. Exerc. Sport Sci. Rev. 18, 29-63.

Stanley, W. C., Gertz, E. W., Wisneski, J. A., Morris, D. L., Neese, R. A., and Brooks, G. A. (1985). Systemic lactate kinetics during graded exercise in man. Am. J. Phys. 249, E595-E602. doi: 10.1152/ajpendo.1985.249.6.E595

Stienen, G. J., Kiers, J. L., Bottinelli, R., and Reggiani, C. (1996). Myofibrillar ATPase activity in skinned human skeletal muscle fibres: fibre type and temperature dependence. J. Physiol. 493, 299-307. doi: 10.1113/jphysiol.1996.sp021384

Stienen, G. J., Zaremba, R., and Elzinga, G. (1995). ATP utilization for calcium uptake and force production in skinned muscle fibres of Xenopus laevis. J. Physiol. 482, 109-122. doi: 10.1113/jphysiol.1995.sp020503

Szentesi, P., Zaremba, R., van Mechelen, W., and Stienen, G. J. (2001). ATP utilization for calcium uptake and force production in different types of human skeletal muscle fibres. J. Physiol. 531, 393-403. doi: 10.1111/ j.1469-7793.2001.0393i.x

Tanner, R. K., Fuller, K. L., and Ross, M. L. (2010). Evaluation of three portable blood lactate analysers: Lactate Pro, Lactate Scout and Lactate Plus. Eur. J. Appl. Physiol. 109, 551-559. doi: 10.1007/s00421-010-1379-9

Tesch, P. A., and Karlsson, J. (1985). Muscle fiber types and size in trained and untrained muscles of elite athletes. J. Appl. Physiol. 59, 1716-1720. doi: 10.1152/jappl.1985.59.6.1716

Vollestad, N. K., and Blom, P. C. (1985). Effect of varying exercise intensity on glycogen depletion in human muscle fibres. Acta Physiol. Scand. 125, 395-405. doi: 10.1111/j.1748-1716.1985.tb07735.x
Wakeling, J. M., Kaya, M., Temple, G. K., Johnston, I. A., and Herzog, W. (2002). Determining patterns of motor recruitment during locomotion. J. Exp. Biol. 205, 359-369.

Wakeling, J. M., Lee, S. S., Arnold, A. S., de Boef Miara, M., and Biewener, A. A. (2012). A muscle's force depends on the recruitment patterns of its fibers. Ann. Biomed. Eng. 40, 1708-1720. doi: 10.1007/s10439-012-0531-6

Wakeling, J. M., Uehli, K., and Rozitis, A. I. (2006). Muscle fibre recruitment can respond to the mechanics of the muscle contraction. J. R. Soc. Interface 3, 533-544. doi: 10.1098/rsif.2006.0113

Weltman, A., Snead, D., Stein, P., Seip, R., Schurrer, R., Rutt, R., et al. (1990). Reliability and validity of a continuous incremental treadmill protocol for the determination of lactate threshold, fixed blood lactate concentrations, and VO2max. Int. J. Sports Med. 11, 26-32. doi: 10.1055/s-2007-1024757

Conflict of Interest: The authors declare that the research was conducted in the absence of any commercial or financial relationships that could be construed as a potential conflict of interest.

Copyright (๑) 2021 Hering and Stepan. This is an open-access article distributed under the terms of the Creative Commons Attribution License (CC BY). The use, distribution or reproduction in other forums is permitted, provided the original author(s) and the copyright owner(s) are credited and that the original publication in this journal is cited, in accordance with accepted academic practice. No use, distribution or reproduction is permitted which does not comply with these terms. 